\title{
Primary bladder amyloidosis mimicking bladder malignancy: a case report and literature review
}

\begin{abstract}
Introduction and objectives: We report a rare case of localized primary bladder amyloidosis, and we conducted a literature review.
\end{abstract}

Materials and methods: A 70-year-old man with gross haematuria and storage bladder symptoms was diagnosed with primary bladder amyloidosis with clinical and radiological features of bladder tumor. A comprehensive review of English literature was done. PubMed, NCBI and Science Direct were searched using the following search terms: "primary"; "Bladder"; "Amyloidosis".

Results: Primary localized bladder amyloidosis is a rare and benign urological disease with approximately 210 reported cases in the English literature. It is usually of the AL type and commonly presents with gross painless haematuria and storage bladder symptoms, mimicking bladder malignancy. Imaging investigations such as ultrasound scan, CT scan and MRI are not diagnostic, whereas histopathological examination confirms the diagnosis. Transurethral resection of the bladder lesion is the treatment of choice; however local recurrence is common ( $50 \%$ of cases).

Conclusion: Localised bladder amyloidosis is almost always of AL type and does not progress to systemic amyloidosis. Treatment is not curative and recurrence is common, hence surveillance cystoscopy is required.

Keywords: bladder lesion, amyloidosis, diagnosis, management
Volume I Issue 2 - 2014

\author{
Salahia MG,' Kebbe Y,' Arora A, ${ }^{2}$ Pinto T, ${ }^{3}$ \\ Hammadeh $\mathrm{MY}^{4}$ \\ 'Lewisham and Greenwich NHS Trust, London \\ ${ }^{2}$ Radiology Department, King's College Hospital NHS Trust, \\ London \\ ${ }^{3}$ Histopathology Department, Lewisham and Greenwich NHS \\ Trust, London \\ ${ }^{4}$ Urology Department, Lewisham and Greenwich NHS Trust, \\ London
}

Correspondence: Salahia MG, Hampstead Mews, South Eden Park Road, Beckenham, Kent, BR33FE, Fax 02086585656,

Email gsalahia@hotmail.com

Received: October 21, 2014 | Published: November 27, 2014
Abbreviations: SAA, serum amyloid aprotein; TTR, transthyretin; CT, computed tomography; MR, magnetic resonance; SAP, serum amyloid p component; TUR, trans urethral resection

\section{Introduction}

Amyloidosis is a rare disease characterized by the deposition of extracellular, hyaline and proteinaceous material in various organs of the body. ${ }^{1}$ It can present as a primary or secondary disorder with systemic or localized manifestations. Primary bladder Amyloidosis is more common than that of the renal pelvis, ureter and urethra. ${ }^{2}$ Approximately, 210 cases of primary bladder amyloidosis have been reported worldwide (in over 22 countries). The largest number of cases was reported in the UK (60 cases). Histological examination is always the cornerstone of diagnosis. A comprehensive literature review from 1966 to 2013 was done, the search terms were: "primary", "bladder", "amyloidosis". We present a rare case of primary bladder amyloidosis, which was difficult to distinguish from a bladder carcinoma as they shared the same clinical presentation, imaging and cystoscopy findings.

\section{Case report}

A 70-year-old Caucasian man presented with a three day history of painless gross haematuria associated with storage lower urinary tract symptoms. He is a non-smoker with no family history of bladder cancer. He has a past history of squamous cell carcinoma of the temple, basal cell carcinoma of the eyebrows, plantar fasciitis, rosacea and solar keratosis. Clinical examination was unremarkable. Mid stream urine microscopy and culture confirmed microscopic haematuria and no significant bacterial growth. Urine cytology showed no malignant cells. He had normal PSA, liver function tests, renal function tests, clotting profile and FBC. Ultrasound scan of the kidneys, ureters and bladder showed a $2 \mathrm{~cm}$ echogenic bladder lesion in the right lateral bladder wall (Figure 1). Cystoscopy showed a $2 \mathrm{~cm}$ calcified bladder lesion on the posterior wall with another $0.5 \mathrm{~cm}$ lesion above the trigone (Figure 2). The appearance was highly suspicious of a solid bladder tumor or bladder schistosomiasis. Trans-urethral resection of these two bladder lesions was performed with deep resection. Complete resection was achieved in the smaller lesion but not in the larger lesion due to the density of the calcifications. Bimanual examination under anaesthesia before and after the bladder lesion resection showed no bladder wall thickness or palpable bladder mass. Histology of the resected bladder lesion ruled out bladder malignancy and demonstrated a degenerative eosinophilic material with extensive ossification and accompanying foreign body giant cell reaction in keeping with bladder amyloidosis (Figure 3).

Follow-up flexible cystoscopy at 4 months under local anaesthesia showed recurrence of the larger, incompletely resected $2 \mathrm{~cm}$ bladder lesion (Figure 4). Subsequent CT scan with contrast showed a lesion on the right bladder wall. The abdominal organs showed no abnormality and there was no abdominal or pelvic lymphadenopathy (Figure 5). A Pelvic MRI showed an enhancing area of thickening on the right bladder wall with ill-defined peri-vesical fat in that area (Figure 6). The patient had a further cystoscopy and complete resection of the bladder lesion was achieved due to less inclusive calcification. Histology confirmed amyloid deposition, with applegreen birefringence, with extensive ossification and accompanying giant cell reaction and no evidence of malignancy. Immunochemical staining of amyloid deposits, using monospecific antibodies reactive with serum amyloid a protein (SAA), transthyretin (TTR) and Kappa and Lambda immunoglobulin chains was negative. Therefore, the conclusion was that the amyloid is of non-AA type. A further follow up with flexible cystoscopy under local anaesthesia every 6months 
showed no recurrence of the amyloid lesion in the bladder up to two years after the second resection (Figure 7).

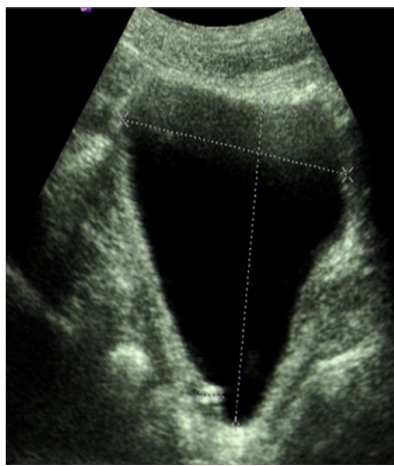

Figure I Bladder ultrasound scan showing echogenic filling defect on the posterior aspect of the bladder.

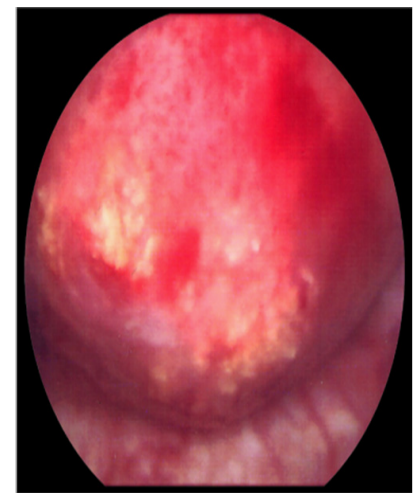

Figure 2 Flexible cystoscopy: $2 \mathrm{~cm}$ calcified bladder lesion on the right wall with another $0.5 \mathrm{~cm}$ small lesion above the trigon.

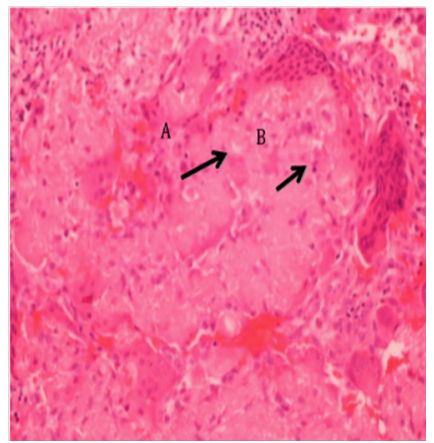

Figure 3 A: Amorphous eosinophilic material (fibrillar protein) in keeping with amyloidosis; B: Foreign body giant cell reaction.

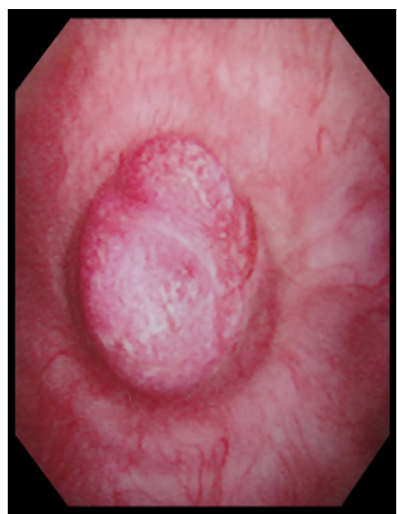

Figure 4 The recurrent lesion had less calcification and had an appearance similar to solid bladder tumor.

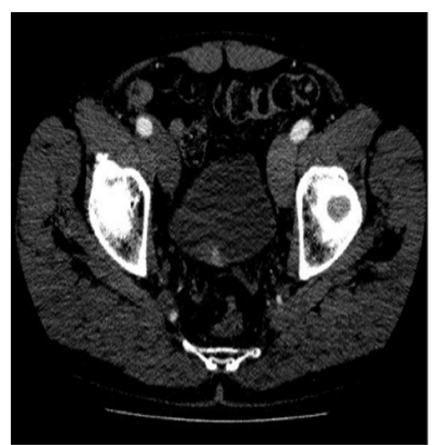

Figure 5 Non-invasive bladder lesion on the right bladder wall on a contrast CT scan.

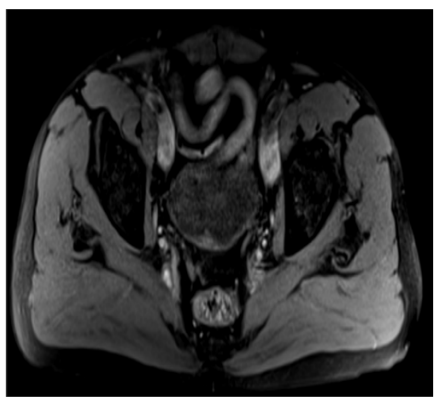

Figure 6 Non-invasive bladder lesion on the right bladder wall on a contrast CT scan.

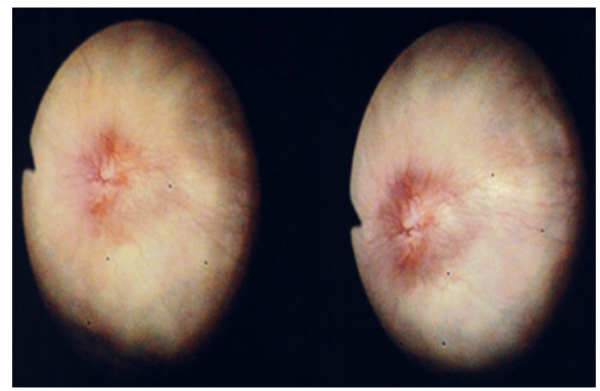

Figure 7 Non-invasive bladder lesion on the right bladder wall on a contrast CT scan.

The patient was referred to the National Amyloid Centre to exclude systemic amyloidosis. SAP scintigraphy ruled out visceral amyloid deposition. ECG, echocardiogram and cardiac biomarkers were normal. Para protein on immunofixation was absent. Serum free light chains and urinary Bence Jones proteins were normal. There was no evidence of clonal plasma cell dyscrasia. It was concluded that this patient had localized primary bladder amyloidosis of non-AA type with no evidence of circulating $\mathrm{M}$ protein or normal free light chains.

\section{Discussion}

Amyloidosis is characterized by the extracellular deposition of the fibrillar protein, amyloid. ${ }^{3}$ It can be localized or systemic and affects both sexes equally. ${ }^{4}$ Each of localized and systemic amyloidosis can be either primary (AL) associated with immunocyte dyscrasia or secondary (AA) as a complication of chronic inflammation. ${ }^{5}$ It can occur anywhere in the urinary tract, starting from kidney to renal pelvis, ureters, urinary bladder, urethra and even penis. ${ }^{6}$ The involvement of the kidney is much more common in secondary amyloidosis, whereas in primary amyloidosis, the urinary bladder is more likely to be the organ of deposition. ${ }^{2}$ Localised bladder amyloidosis is almost always of AL primary type and is unlikely to progress to systemic amyloidosis. Gross haematuria was the 
presenting symptom in $60 \%$ of the cases, whereas $20 \%$ of patients presented with storage bladder symptoms, and $20 \%$ with both. ${ }^{7}$ Our case presented with frank painless haematuria and longstanding storage lower urinary tract symptoms. Primary bladder amyloidosis usually does not have significant findings on clinical examination or on routine laboratory tests. Computed tomography (CT) and magnetic resonance (MR) imaging depict focal or, less commonly, diffuse thickening of the bladder wall or a filling defect in the bladder lumen that are non-diagnostic. ${ }^{8}$ On cystoscopy, amyloidosis poses a diagnostic dilemma since it presents as an irregular, tumor-like, easily bleeding, calcified lesion. ${ }^{1}$ It can be seen also as nodular or polypoidal single or multiple lesions. This appearance raises the suspicion of neoplasia, schistomiasis, hemorrhagic cystitis, interstitial cystitis and tuberculosis; thus, a biopsy of the lesion is mandatory. Diagnosis is based on histological examination. ${ }^{9}$ A Congo red stain examined under polarized light shows characteristic apple-green birefringence as described by Dirvy in 1927.

Investigations to rule out systemic amyloidosis should be performed. These include rectal and abdominal fat biopsies, urinary Bence Jones proteins, serum electrophoresis, and serum amyloid $\mathrm{P}$ component (SAP) scintigraphy that usually shows specific uptake in organs such as liver, spleen, kidney, adrenals, etc. ${ }^{10}$ Localised amyloidosis generally does not require systemic therapy. ${ }^{2}$ Treatment is usually transurethral resection (TUR) of the lesion, the outcome of which is successful in most cases. Isolated reports of medical regimens e.g. oral colchicine, cepharanthin, nitrofurazone and intravesical installation of dimethylsulphoxide as primary or adjuvant therapy show only limited success. ${ }^{3}$ Rarely, where the affected area is very large and there is severe haematuria, radical (partial or total) cystectomy can be performed..$^{11}$ Robotic laparoscopic prostate-sparing simple cystectomy with intra corporeal neo bladder and preservation of the seminal vesicles and vas deferens has been recently suggested as a potential treatment. ${ }^{12}$

Recurrence may occur within a few months to several years in $50 \%$ of cases. ${ }^{13}$ Hence, patients may require lifelong surveillance. ${ }^{14}$ Recurrent lesions require re-resection. To our knowledge, there has been no reported recurrence of amyloidosis in all open-treated cases regardless of total or partial cystectomy. Amyloidosis is a benign non-neoplastic lesion that does not exhibit causal relationship with urothelial carcinoma. There are only three reported cases in the literature where unrelated bladder cancer developed in patients with primary bladder amyloidosis monitored over a long period. ${ }^{15}$ Nevertheless, regular and long- term follow-up with cystoscopy is indicated for recurrent amyloidosis.

\section{Acknowledgements}

None.

\section{Conflict of interest}

The author declares no conflict of interest.

\section{References}

1. DeSouza MA, Rekhi B, Thyavihally YB, et al. Localized amyloidosis of the urinary bladder, clinically masquerading as bladder cancer. Indian $J$ Pathol Microbiol. 2008;51(3):415-417.

2. Kawashima A, Alleman WG, Takahashi N, et al. Imaging Evaluation of Amyloidosis of the Urinary Tract and Retroperitoneum. Radiographics. 2011;31(6):1569-1582.

3. Mark I R, Goodlad J, Lloyd-Davies RW. Localized amyloidosis of the genito-urinary tract. J R Soc Med. 1995;88(6):320-324.

4. Wilkinson M, Fanning DM, Flood H. Primary bladder amyloidosis. BMJ Case Reports. 2011;2011:bcr.05.2011.4211.

5. Jain M, Kumari N, Chhabra $P$, et al. Localized amyloidosis of urinary bladder: A diagnostic dilemma. Indian J Pathol Microbiol. 2008;51(2):247-249

6. Altwairgi A. Primary amyloidosis of the urinary bladder presenting as painless heamaturia. Int J Health Sci (Qassim). 2011;5(2):181-185.

7. Malek RS, Wahner-Roedler DL, Gertz MA, et al. Primary Localized Amyloidosis of The bladder: Experience with Dimethyl Sulfoxide Therapy. J Urol. 2002;168(3):1018-1020.

8. Urban BA, Fishman EK, Goldman SM, et al. CT evaluation of amyloidosis: spectrum of disease. Radiographics. 1993;13(6):12951308.

9. Lee HL, Chang SG, Yang MH, et al. Primary Localized Amyloidosis of the Bladder-A case report. J Korean Med Sci. 1994;9(3):273-275.

10. Goswami AK, Vaidyanathan S, Rao MS, et al. Primary localised amyloidosis of urinary bladder (a case report). J Postgrad Med. 1984;30(4):253-254

11. Yalcin V, Citgez S, Agaoglu I. Localised primary amyloidosis of the urinary bladder: case series of two patients. BMJ Case Rep. 2009;2009:1367.

12. Hosseini A, Ploumidis A, Adding C, et al. Radical surgery for treatment of primary localized bladder amyloidosis: Could prostate-sparing robot-assisted cystectomy with intracorporeal urinary diversion be an option? Scand J Urol. 2013;47(1):72-75.

13. Ferch R, Haskell R, Farebrother T. Primary amyloidosis of urinary bladder and ureter. Br J Urol. 1997;80(6):953-954.

14. Agarwal SK, Walmsley, Marley NJ. Primary amyloidosis of urinary bladder. J R Soc Med. 1995;88(3):171-172.

15. Gupta P, Hanamshetti S, Kulkarni JN. Primary amyloidosis with high grade transitional cell carcinoma of bladder: A rare case report. $J$ Cancer Res Ther. 2012;8(2):297-299. 